\title{
Searching for a Target Whose Truncated Brownian Motion
}

\author{
Abd Elmoneim A. Teamah, Mohamed A. El-Hadidy, Marwa M. El-Ghoul \\ Department of Mathematics, Faculty of Science, Tanta University, Tanta, Egypt \\ Email: teamah4@hotmail.com,melhadidi@science.tanta.edu.eg,marwa_mabrouk@science.tanta.edu.eg
}

How to cite this paper: Teamah, A.A., El-Hadidy, M.A. and El-Ghoul, M.M. (2017) Searching for a Target Whose Truncated Brownian Motion. Applied Mathematics, 8, 786-798.

https://doi.org/10.4236/am.2017.86061

Received: March 10, 2017

Accepted: June 11, 2017

Published: June 14, 2017

Copyright $\odot 2017$ by authors and Scientific Research Publishing Inc. This work is licensed under the Creative Commons Attribution International License (CC BY 4.0).

http://creativecommons.org/licenses/by/4.0/ (c) (i) Open Access

\begin{abstract}
This paper presents search model for a randomly moving target which follows truncated Brownian motion. The conditions that make the expected value of the first meeting time between the searcher and the target is finite are given. We show the existence of an optimal strategy which minimizes this first meeting time.
\end{abstract}

\section{Keywords}

Search Plan, Truncated Brownian Motion, Expected Value,

First Meeting Time

\section{Introduction}

Detecting the holes on the oil pipeline under water prevents without a disaster such as that occurred in the Guilf of Mexico on April 2010. Linear search model is the one of the interesting search models which is used to detect these holes.

Searching for a Brownian target on the real line has been studied by El-Rayes et al. [1]. They illustrated this problem when the searcher started the searching process from the origin. They found the conditions that make the expected value of the first meeting time between the searcher and target is finite. They showed the existence of the optimal search plan which makes the expected value of the first meeting time between the searcher and target minimum. Mohamed et al. [2] studied this problem for a Brownian target on one of $n$-intersected real lines. The information about the target position is not available to the searchers at all the time. Recently, El-Hadidy [3], studied this search problem for a $d$-dimensional Brownian target that moves randomly on $d$-space.

The main contribution of this paper centers on studying the search problem for a one-dimensional truncated Brownian motion. The searcher moves with a linear motion. This kind of search problems recently has various applications in 
physics such as finding a very small object that moves in the space like viruses and bacteria, or the object that is very large, like stars and planets. We aim to show the conditions that make the expected value of the first meeting time between the searcher and target is finite and show the existence of the optimal search plan that minimizes it.

This paper is organized as follows. In Section 2, we introduce the problem. In Section 3, the finite search plan and the expected value of the first meeting are discussed. In Section 4, we find the existence of optimal search path. In Section 5 , we give an application to calculate the expected value of the first meeting time between the searcher and target.

\section{Problem Formulation}

The problem under study can be formally described as follows: We have a searcher starts the searching process from the origin of the line. The searcher moves continuously along its line in both directions of the starting point. The searcher would conduct its search in the following manner: Start at $H_{0}=0$ and go to the left (right) as far as $H_{1}$. Then, turn back to explore the right (left) part of $H_{0}=0$ as far as $H_{2}$. Retrace the steps again to explore the left (right) part of $H_{1}$ as far as $H_{3}$ and so on. The target is assumed to move randomly on the real line according to the one-dimensional truncated Brownian motion. The initial position of the target is unknown but the searcher knows the probability distribution of it, i.e., the probability distribution of the target is given at time 0 , and the process $\left\{W(t), t \in R^{+}\right\}$, which controls the target's motion, is truncated Brownian motion, where it has stationary independent increments, for any time interval $\left(t_{1}, t_{2}\right) W\left(t_{1}\right)-W\left(t_{2}\right)$ follows truncated normally distributed, and this process is called a truncated Brownian motion with drift $\mu^{\prime}$ and variance $\sigma^{\prime 2}$. A search plan with speed $V$, which the searcher follows it, is a function

$\phi: R^{+} \rightarrow R$ such that:

$\left|\phi\left(t_{1}\right)-\phi\left(t_{2}\right)\right| \leq V\left|t_{1}-t_{2}\right|, \forall t_{1}, t_{2} \in R^{+}$. where $R$ is the set of real numbers

And $V$ is a constant in $R^{+}$and $\phi(0)=0$. The first meeting time $\tau_{\phi}$ is a random variable valued in $R^{+}$defined as:

$$
\tau_{\phi}=\inf \left\{t, \phi(t)=X_{0}+W(t)\right\}
$$

where $X_{0}$ is a random variable follows truncated normal distribution and independent with $W(t)$ and represent initial position of the target. The aim of the searcher is to minimize the expected value of $\tau_{\phi}$.

Let $\Phi_{V}(t)$ is the set of all search plans with speed $V$. The problem is to find a search plan $\phi \in \Phi_{V}(t)$ such that $E \tau_{\phi}<\infty$, in this case we call $\phi$ is a finite search plan if:

$$
E \tau_{\phi^{*}} \leq E \tau_{\phi}, \forall \phi \in \Phi_{V}(t) .
$$

Then we call $\phi^{*}$ is optimal search plan.

Let $\lambda$ and $\theta$ be positive integers greater than one and $v$ be a rational number such that:

1) $v>\left|\mu^{\prime}\right|$. 
2) $\theta>1$ such that $c=\frac{v(\theta-1)}{(\theta+1)}>\left|\mu^{\prime}\right|$.

We shall define two sequences $\left\{G_{i}\right\}_{i \geq 0},\left\{H_{i}\right\}_{i \geq 0}$ and a search plan with speed $\mathrm{v}$ as follows:

$$
G_{i}=\lambda\left(\theta^{i}-1\right), H_{i}=(-1)^{i+1} c\left[G_{i}+1+(-1)^{i+1}\right] .
$$

For any $t \in R^{+}$, if $G_{i} \leq t \leq G_{i+1}, \phi(t)=H_{i}+(-1)^{i}\left(t-G_{i}\right) v$.

Note that the truncated normal distribution:

If $Y$ is $N\left(\mu, \sigma^{2}\right)$ then, the probability density function of double truncated of $X$ is given by:

$$
f_{X}(x)=\frac{\frac{1}{\sqrt{2 \pi \sigma^{2}}} \exp \left(\frac{-(x-\mu)^{2}}{2 \sigma^{2}}\right)}{F\left(\frac{b-\mu}{\sigma}\right)-F\left(\frac{a-\mu}{\sigma}\right)} I_{[a, b]}(x) \text { for } a \leq x \leq b .
$$

where $F$ is the cumulative distribution function and $I_{[a, b]}(x)=1$ which is the indicator function.

And the expected value for truncated normal distribution is given by:

$$
\mu^{\prime}=E(x)=\mu+\left[\frac{f\left(\frac{a-\mu}{\sigma}\right)-f\left(\frac{b-\mu}{\sigma}\right)}{F\left(\frac{b-\mu}{\sigma}\right)-F\left(\frac{a-\mu}{\sigma}\right)}\right] \sigma .
$$

The variance for truncated normal distribution is given by:

$$
\begin{aligned}
\sigma^{\prime 2}=\operatorname{var}(x)= & \sigma^{2}\left[1+\frac{\left(\frac{a-\mu}{\sigma}\right) f\left(\frac{a-\mu}{\sigma}\right)-\left(\frac{b-\mu}{\sigma}\right) f\left(\frac{b-\mu}{\sigma}\right)}{F\left(\frac{b-\mu}{\sigma}\right)-F\left(\frac{a-\mu}{\sigma}\right)}\right] \\
& -\sigma^{2}\left[\frac{f\left(\frac{a-\mu}{\sigma}\right)-f\left(\frac{b-\mu}{\sigma}\right)}{F\left(\frac{b-\mu}{\sigma}\right)-F\left(\frac{a-\mu}{\sigma}\right)}\right]
\end{aligned}
$$

\section{Existence of a Finite Search Plan}

In this section we aim to find the conditions that make the search plan to be finite and minimize the expected value of the first meeting time.

Theorem 3.1: Let $v$ be the measure defined on $R$ by $X_{o}$ and if $\phi(t)$ is the search plan defined above, then the expectation $E_{\tau_{\phi}}$ is finite if:

$$
\int_{a}^{0} \sum_{i=1}^{\infty} \theta^{2 i} p\left\{\tilde{\psi}\left(G_{2 i}\right)<-x\right\} v(\mathrm{~d} x), \int_{0}^{b} \sum_{i=1}^{\infty} \theta^{2 i+1} p\left\{\psi\left(G_{2 i+1}\right)>-x\right\} v(\mathrm{~d} x)
$$

are finite, where $\tilde{\psi}\left(G_{2 i}\right)=W\left(G_{2 i}\right)+c G_{2 i}, \psi\left(G_{2 i+1}\right)=W\left(G_{2 i+1}\right)-c\left(G_{2 i+1}\right)$.

Proof:

The continuity of $\phi(t)$ and $W(t)$ imply that if $X_{0}$ is positive then $X_{0}+W(t)$ is greater than $\phi(t)$ until the first meeting, also if $X_{0}$ is negative then $X_{0}+W(t)$ is smaller than $\phi(t)$ until the first meeting, hence for any 
$i \geq 0$

$$
\begin{aligned}
p\left(\tau_{\phi}>G_{2 i+1}\right) \leq & \int_{a}^{0} p\left(X_{0}+W\left(G_{2 i}\right)<H_{2 i} / X_{0}=x\right) v(\mathrm{~d} x) \quad \text { where } a<0, b>0 . \quad(1) \\
& +\int_{0}^{b} p\left(X_{0}+W\left(G_{2 i+1}\right)>H_{2 i+1} / X_{0}=x\right) v(\mathrm{~d} x),
\end{aligned}
$$

Using the notation: $\tilde{\psi}\left(G_{2 i}\right)=W\left(G_{2 i}\right)+c G_{2 i}$, we obtain:

$W\left(G_{2 i}\right)-H_{2 i}<X_{0}=-\mathrm{x}$, then $W\left(G_{2 i}\right)-\left[(-1)^{2 i+1} c\left(G_{2 i}+1+(-1)^{2 i+1}\right)\right]<X_{0}=-x$.

Leads to: $W\left(G_{2 i}\right)-(-1) c\left[G_{2 i}+1-1\right]=W\left(G_{2 i}\right)+c G_{2 i}=\tilde{\psi}\left(G_{2 i}\right)<-\chi$.

Similarly, by using the notation: $\psi\left(G_{2 i+1}\right)=W\left(G_{2 i+1}\right)-c G_{2 i+1}$.

$$
p\left(\tau_{\phi}>G_{2 i+1}\right) \leq \int_{a}^{0} p\left(\tilde{\psi}\left(G_{2 i}\right)<-x\right) v(\mathrm{~d} x)+\int_{0}^{b} p\left(\psi\left(G_{2 i+1}\right)>-x\right) v(\mathrm{~d} x) .
$$

Similarly for any $i>0$

$$
p\left(\tau_{\phi}>G_{2 i}\right) \leq \int_{a}^{0} p\left(\tilde{\psi}\left(G_{2 i}\right)<-x\right) v(\mathrm{~d} x)+\int_{0}^{b} p\left(\psi\left(G_{2 i-1}\right)>-x\right) v(\mathrm{~d} x) .
$$

But we have

$$
\begin{aligned}
E\left(\tau_{\phi}\right) & =\int_{0}^{\infty} p\left(\tau_{\phi}>t\right) \mathrm{d} t=\sum_{i=0}^{\infty} \int_{G_{i}}^{G_{i+1}} p\left(\tau_{\phi}>t\right) \mathrm{d} t \\
& \leq \sum_{i=0}^{\infty} \int_{G_{i}}^{G_{i+1}} p\left(\tau_{\phi}>G_{i}\right) \mathrm{d} t=\sum_{i=0}^{\infty}\left(G_{i+1}-G_{i}\right) p\left(\tau_{\phi}>G_{i}\right) .
\end{aligned}
$$

Since $G_{i}=\lambda\left(\theta^{i}-1\right)$ and $G_{i+1}=\lambda\left(\theta^{i+1}-1\right)$

$$
\begin{aligned}
& G_{i+1}-G_{i}=\lambda\left(\theta^{i+1}-1\right)-\lambda\left(\theta^{i}-1\right)=\lambda\left(\theta^{i+1}-\theta^{i}\right) \\
& E\left(\tau_{\phi}\right) \leq \sum_{i=0}^{\infty} \lambda \theta^{i}(\theta-1) p\left(\tau_{\phi}>G_{i}\right)=\lambda(\theta-1) \sum_{i=0}^{\infty} \theta^{i} p\left(\tau_{\phi}>G_{i}\right) \\
&= \lambda(\theta-1)\left[p\left(\tau_{\phi}>0\right)+\theta p\left(\tau_{\phi}>G_{1}\right)+\theta^{2} p\left(\tau_{\phi}>G_{2}\right)+\theta^{3} p\left(\tau_{\phi}>G_{3}\right)+\cdots\right] \\
&= \lambda(\theta-1)\left[p\left(\tau_{\phi}>0\right)+\theta p\left(\tau_{\phi}>G_{1}\right)\right. \\
&+\theta^{2}\left\{\int_{a}^{0} p\left(\tilde{\psi}\left(G_{2}<-x\right)\right) v(\mathrm{~d} x)+\int_{0}^{b} p\left(\psi\left(G_{1}>-x\right)\right) v(\mathrm{~d} x)\right\} \\
&+\theta^{3}\left\{\int_{a}^{0} p\left(\tilde{\psi}\left(G_{2}<-x\right)\right) v(\mathrm{~d} x)+\int_{0}^{b} p\left(\psi\left(G_{3}>-x\right)\right) v(\mathrm{~d} x)\right\} \\
&+\theta^{4}\left\{\int_{a}^{0} p\left(\tilde{\psi}\left(G_{4}<-x\right)\right) v(\mathrm{~d} x)+\int_{0}^{b} p\left(\psi\left(G_{3}>-x\right)\right) v(\mathrm{~d} x)\right\}+\cdots \\
&+\theta^{k}\left\{\int_{a}^{0} p\left(\tilde{\psi}\left(G_{k}<-x\right)\right) v(\mathrm{~d} x)+\int_{0}^{b} p\left(\psi\left(G_{k-1}>-x\right)\right) v(\mathrm{~d} x)\right\} \\
&+\theta^{k+1}\left\{\int_{a}^{0} p\left(\tilde{\psi}\left(G_{k}<-x\right)\right) v(\mathrm{~d} x)+\int_{0}^{b} p\left(\psi\left(G_{k+1}>-x\right)\right) v(\mathrm{~d} x)\right\} \\
&+\theta^{k+2}\left\{\int_{a}^{0} p\left(\tilde{\psi}\left(G_{k+2}<-x\right)\right) v(\mathrm{~d} x)+\int_{0}^{b} p\left(\psi\left(G_{k+1}>-x\right)\right) v(\mathrm{~d} x)\right\} \\
&\left.+\theta^{k+3}\left\{\int_{a}^{0} p\left(\tilde{\psi}\left(G_{k+2}<-x\right)\right) v(\mathrm{~d} x)+\int_{0}^{b} p\left(\psi\left(G_{k+3}>-x\right)\right) v(\mathrm{~d} x)\right\}+\cdots\right] .
\end{aligned}
$$


Then:

$$
\begin{aligned}
E\left(\tau_{\varphi}\right) \leq & \lambda(\theta-1)\left[p\left(\tau_{\phi}>0\right)+\theta p\left(\tau_{\phi}>G_{1}\right)+\theta^{2} \int_{a}^{0} p\left(\tilde{\psi}\left(G_{2}\right)<-x\right) v(\mathrm{~d} x)\right. \\
& +\theta^{2} \int_{0}^{b} p\left(\psi\left(G_{1}\right)>-x\right) v(\mathrm{~d} x)+\theta^{3} \int_{a}^{0} p\left(\tilde{\psi}\left(G_{2}\right)<-x\right) v(\mathrm{~d} x) \\
& \left.+\theta^{3} \int_{0}^{b} p\left(\psi\left(G_{3}\right)>-x\right) v(\mathrm{~d} x)+\cdots\right] \\
= & \lambda(\theta-1)\left[p\left(\tau_{\phi}>0\right)+\theta p\left(\tau_{\phi}>G_{1}\right)+\theta^{2} \int_{0}^{b} p\left(\psi\left(G_{1}\right)>-x\right) v(\mathrm{~d} x)\right. \\
& +\left(\theta^{2}+\theta^{3}\right) \int_{a}^{0} p\left(\tilde{\psi}\left(G_{2}\right)<-x\right) v(\mathrm{~d} x)+\left(\theta^{3}+\theta^{4}\right) \int_{0}^{b} p\left(\psi\left(G_{3}\right)>-x\right) v(\mathrm{~d} x) \\
+ & \left(\theta^{4}+\theta^{5}\right) \int_{a}^{0} p\left(\tilde{\psi}\left(G_{4}\right)<-x\right) v(\mathrm{~d} x)+\cdots+\left(\theta^{k}+\theta^{k+1}\right) \int_{a}^{0} p\left(\tilde{\psi}\left(G_{k}\right)<-x\right) v(\mathrm{~d} x) \\
+ & \left.\left(\theta^{k+1}+\theta^{k+2}\right) \int_{0}^{b} p\left(\psi\left(G_{k+1}\right)>-x\right) v(\mathrm{~d} x)+\cdots\right] .
\end{aligned}
$$

Then:

$$
\begin{aligned}
E\left(\tau_{\phi}\right) \leq & \lambda(\theta-1)\left[p\left(\tau_{\phi}>0\right)+\theta p\left(\tau_{\phi}>G_{1}\right)+\theta^{2} \int_{0}^{b} p\left(\psi\left(G_{1}\right)>-x\right) v(\mathrm{~d} x)\right. \\
& +\theta^{2}(\theta+1) \int_{a}^{0} p\left(\tilde{\psi}\left(G_{2}\right)<-x\right) v(\mathrm{~d} x)+\theta^{4}(\theta+1) \int_{a}^{0} p\left(\tilde{\psi}\left(G_{4}\right)<-x\right) v(\mathrm{~d} x) \\
& +\cdots+\theta^{k}(\theta+1) \int_{a}^{0} p\left(\tilde{\psi}\left(G_{k}\right)<-x\right) v(\mathrm{~d} x)+\theta^{3}(\theta+1) \int_{0}^{b} p\left(\psi\left(G_{3}\right)>-x\right) v(\mathrm{~d} x) \\
& \left.+\theta^{5}(\theta+1) \int_{0}^{b} p\left(\psi\left(G_{5}\right)>-x\right) v(\mathrm{~d} x)+\cdots+\theta^{k+1}(\theta+1) \int_{0}^{b} p\left(\psi\left(G_{k+1}\right)>-x\right) v(\mathrm{~d} x)+\cdots\right]
\end{aligned}
$$

Leads to:

$$
E\left(\tau_{\phi}\right) \leq \lambda(\theta-1)\left[g+(\theta+1)\left(\int_{a}^{0} M(x) v(\mathrm{~d} x)+\int_{0}^{b} B(x) v(\mathrm{~d} x)\right)\right] .
$$

where:

$$
\begin{gathered}
g=p\left(\tau_{\phi}>0\right)+\theta p\left(\tau_{\phi}>G_{1}\right)+\theta^{2} \int_{0}^{b} p\left(\psi\left(G_{1}\right)>-x\right) v(\mathrm{~d} x) \\
M(x)=\sum_{i=1}^{\infty} \theta^{2 i} p\left(\tilde{\psi}\left(G_{2 i}\right)<-x\right) . \\
B(x)=\sum_{i=1}^{\infty} \theta^{2 i+1} p\left(\psi\left(G_{2 i+1}\right)>-x\right) .
\end{gathered}
$$

Lemma 3.1: Let $a_{n} \geq 0$ for $n \geq 0$, and $a_{n+1} \leq a_{n}$. Let $\left\{d_{n}\right\}, n \geq 0$ be a strictly increasing sequence of integers with $d_{0}=0$. Then for any $k \geq 0$ see [4]

$$
\sum_{n=k}^{\infty}\left(d_{n+1}-d_{n}\right) a_{d_{n+1}} \leq \sum_{n=d_{k}}^{\infty} a_{k} \leq \sum_{n=k}^{\infty}\left(d_{n+1}-d_{n}\right) a_{d_{n}} .
$$


Lemma 3.2: If $c \geq \mu^{\prime}$, where $\mu^{\prime}$ is the drift of $W(t)$ and $c$ is a constant, then for any $t>0$, and for some $\varepsilon>0$

$$
p\{W(t) \geq c t\} \leq \frac{1}{2} \frac{1}{F(b)-F(a)} \varepsilon^{t} .
$$

Proof:

$$
\begin{aligned}
p\{W(t) \geq c t\} & =p\left\{\sigma^{\prime} \sqrt{t} X+\mu^{\prime} \geq c t\right\}=p\left\{X \geq \frac{\left(c-\mu^{\prime}\right) t}{\sigma^{\prime} \sqrt{t}}\right\} \\
& =\int_{k^{\prime}}^{b} \frac{1}{\sqrt{2 \pi}} \exp \left(\frac{-x^{2}}{2}\right) \\
F(b)-F(a) & \mathrm{d} x, \quad k^{\prime}=\frac{\left(c-\mu^{\prime}\right) t}{\sigma^{\prime} \sqrt{t}} .
\end{aligned}
$$

Hence:

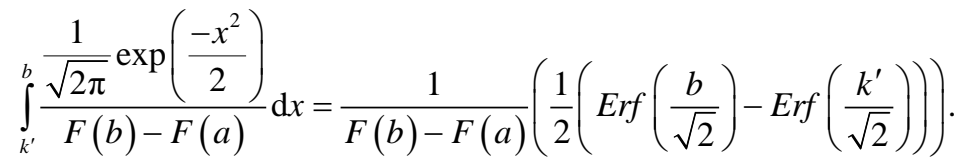

And then

$\int_{k}^{\infty} \frac{1}{\sqrt{2 \pi}} \exp \left(\frac{-x^{2}}{2}\right) \mathrm{d} x=\frac{1}{2} \operatorname{ErfC}\left(\frac{k}{\sqrt{2}}\right) \leq \frac{1}{2} \varepsilon^{t}$, see [1].

where: $\operatorname{Erfc}$ is the complementary Error function commonly donated $\operatorname{Erfc}(x)$, is an entire function defined by

$$
\begin{aligned}
\operatorname{Erfc}(x)=1-\operatorname{Erf}(x)=\frac{2}{\sqrt{\pi}} \int_{x}^{\infty} \mathrm{e}^{-r^{2}} \mathrm{~d} r, \text { then } \operatorname{Erfc}\left(\frac{k}{\sqrt{2}}\right)=1-\operatorname{Erf}\left(\frac{k}{\sqrt{2}}\right) \\
\begin{aligned}
\frac{1}{2}\left(\operatorname{Erf}\left(\frac{b}{\sqrt{2}}\right)-\operatorname{Erf}\left(\frac{k^{\prime}}{\sqrt{2}}\right)\right) & =\frac{1}{2}\left(1-\operatorname{Erfc}\left(\frac{b}{\sqrt{2}}\right)-1+\operatorname{Erfc}\left(\frac{k^{\prime}}{\sqrt{2}}\right)\right) \\
& =\frac{1}{2}\left(\operatorname{Erfc}\left(\frac{k^{\prime}}{\sqrt{2}}\right)-\operatorname{Erfc}\left(\frac{b}{\sqrt{2}}\right)\right) \\
& \leq \frac{1}{2} \operatorname{Erfc}\left(\frac{k}{\sqrt{2}}\right) \leq \frac{1}{2} \varepsilon^{t} .
\end{aligned}
\end{aligned}
$$

Then:

Then: $\frac{1}{F(b)-F(a)} \frac{1}{2}\left(\operatorname{Erfc}\left(\frac{k^{\prime}}{\sqrt{2}}\right)-\operatorname{Erfc}\left(\frac{b}{\sqrt{2}}\right)\right) \leq \frac{1}{2} \frac{1}{F(b)-F(a)} \varepsilon^{t}$.

Hence: $\quad p\{W(t) \geq c t\} \leq \frac{1}{2(F(b)-F(a))} \varepsilon^{t}$.

where $\varepsilon=\mathrm{e}^{-\frac{(c-\mu)^{2}}{2 \sigma^{2}}}$, and $X$ follows truncated standardized normal distribution.

Lemma 3.3: If $\mu^{\prime} \neq 0, x_{1} \geq x_{2}, t \geq \max \left(\frac{x_{1}}{\mu^{\prime}}, \frac{x_{2}}{\mu^{\prime}}\right), a<0, b>0$ then $p\left\{x_{2} \leq W(t) \leq x_{1}\right\}$ is non-increasing with $t$.

Proof: Since

$$
\begin{gathered}
P\left(x_{2} \leq W(t) \leq x_{1}\right)=P\left(x_{2} \leq \sigma^{\prime} \sqrt{t} X+\mu^{\prime} t \leq x_{1}\right)=P\left(\frac{x_{2}-\mu^{\prime} t}{\sigma^{\prime} \sqrt{t}} \leq X \leq \frac{x_{1}-\mu^{\prime} t}{\sigma^{\prime} \sqrt{t}}\right) \text { if } \\
\mu^{\prime}<0 \text {, then }\left(x_{2}-\mu^{\prime} t\right) / \sigma^{\prime} \sqrt{t} \text { and } \frac{\mathrm{d}}{\mathrm{d} t}\left(\left(x_{2}-\mu^{\prime} t\right) / \sigma^{\prime} \sqrt{t}\right) \geq 0 \text { this implies that }
\end{gathered}
$$


$P\left(x_{2} \leq W(t) \leq x_{1}\right)$ is non-increasing also if $\mu^{\prime}>0$, then $\left(x_{1}-\mu^{\prime} t\right) / \sigma^{\prime} \sqrt{t} \leq 0$, this implies that $P\left(x_{2} \leq W(t) \leq x_{1}\right)$ is non-increasing.

Lemma 3.4: If $W(n)=\sum_{i=1}^{n} X_{i}, n \geq 1$, where $\left\{X_{i}\right\}$ is a sequence of independent identically distributed random variables (i.i.d.r.v), such that $X_{i}$ is truncated normally distributed with parameters $\mu^{\prime}-c$ and $\sigma^{\prime 2}$, and so

$$
q(j, j+1)=\sum_{n=0}^{\infty} p\{-(j+1)<W(n)<-j\}
$$

Satisfies the conditions of the Renewal theorem, see [5].

Theorem 3.2: The chosen search plan satisfies:

$M(x) \leq \tilde{L}(|x|)$ and $B(x) \leq L(|x|)$.

where $L(|x|)$ and $\tilde{L}(|x|)$ are linear functions.

Proof If $x \leq 0$, then $B(x) \leq B(0)$, but we have for $x>0$

$$
B(0)=\sum_{i=1}^{\infty} \theta^{2 i+1} p\left(\psi\left(G_{2 i+1}\right)>0\right), \quad B(x)=\sum_{i=1}^{\infty} \theta^{2 i+1} p\left(G_{2 i+1}>-x\right) .
$$

Then $B(x)=B(0)+\sum_{i=1}^{\infty} \theta^{2 i+1} p\left(-x<\psi\left(G_{2 i+1}\right) \leq 0\right)$.

Lemma 3.2 states that $p\{W(t) \geq c t\} \leq \frac{1}{2} \frac{1}{F(b)-F(a)} \varepsilon^{t}$, then

$$
p(W(t)-c t \geq 0) \leq \frac{1}{2} \frac{1}{F(b)-F(a)} \varepsilon^{t} \Rightarrow p(\psi(t) \geq 0) \leq \frac{1}{2} \frac{1}{F(b)-F(a)} \varepsilon^{t} .
$$

where $\psi(t)=W(t)-c t, \quad B(0)<\frac{1}{F(b)-F(a)} \sum_{i=1}^{\infty} \theta^{2 i+1} \varepsilon^{G_{2 i+1}}, \quad 0<\varepsilon<1$.

We define the following:

1) $\psi(n)=\sum_{i=1}^{n} y_{i}$, where $\left\{y_{i}\right\}_{i \geq 1}$ is a sequence of (i.i.d.r.v), $y_{i} \approx N\left(\mu^{\prime}-c, \sigma^{\prime 2}\right)$

2) $d_{n}=G_{2 n+1}=\lambda\left(\theta^{2 n+1}-1\right)$.

3) we choose $d_{m}$ such that $d_{m}=\max (0,-x / \mu)$, refer to Lemma 3.3 putting $x_{1}=0$ and $x_{2}=-x_{1}$.

4) $a(n)=p(-x<\psi(n) \leq 0)=\sum_{j=0}^{|x|} p(-(j+1)<\psi(n) \leq-j)$.

5) $\alpha=\theta^{2} / \lambda\left(\theta^{2}-1\right)$.

6) $U(j, j+1)=\sum_{n=0}^{\infty} p(-(j+1)<\psi(n) \leq-j)$.

If $n>d_{m}$ then by Lemma 3.3, $a(n)$ is non-increasing and we can apply Lemma 3.1 we obtain;

$$
\begin{aligned}
B(x)-B(0) & =\sum_{n=1}^{m} \theta^{2 n+1} a\left(d_{n}\right)+\alpha \sum_{n=m+1}^{\infty}\left(d_{n}-d_{n-1}\right) a\left(d_{n}\right) \\
& \leq \sum_{n=1}^{m} \theta^{2 n+1}+\alpha \sum_{n=d_{m}}^{\infty} a(n) \leq \frac{\theta^{3}\left(\theta^{2 m}-1\right)}{\left(\theta^{2}-1\right)}+\sum_{j=0}^{|x|} U(j, j+1)
\end{aligned}
$$

$U(j, j+1)$ satisfies the conditions of Renewal theorem (by Lemma 3.4), hence $U(j, j+1)$ is bounded for all $j$, by a constant, so 


$$
B(x) \leq B(0)+S_{1}+S_{2}|x|=L(|x|) .
$$

We can prove $M(x) \leq \tilde{L}(|x|)$ by similar way.

\section{Lemma 3.5:}

$$
E|W(T)| \leq \sigma^{\prime} \sqrt{E(T)}+\left|\mu^{\prime}\right| E(T) .
$$

where $E$ stand for the expectation value and $\sigma^{\prime 2}$ is the variance.

\section{Proof}

Let $X(t)$ be a standard truncated Brownian motion, assume that $T_{n}=\min (T, n)$ is a bounded stopping time $x(t)$. since $x^{2}(t)-t$ is a continuous martingale, then $E\left(X^{2}\left(T_{n}\right)-T_{n}\right)=E X^{2}(0)=0$ see [5]. lemma

But $E\left(X^{2}\left(T_{n}\right)\right)=E\left(\liminf _{n \rightarrow \infty}\left(X^{2}\left(T_{n}\right)\right)\right) \leq\left(\liminf _{n \rightarrow \infty} E\left(X^{2}\left(T_{n}\right)\right)\right)$ by Fatou

$$
\leq\left(\liminf _{n \rightarrow \infty} E\left(X\left(T_{n}\right)\right)\right) .
$$

Hence $E\left(X^{2}(T)\right) \leq E(T)$ by monotonically of $T_{n}$, since $W(t)=\sigma^{\prime} X(t)+\mu^{\prime} t$, then

$$
E|W(T)| \leq \sigma^{\prime} E|X(T)|+\left|\mu^{\prime}\right| E(T) .
$$

But $E|X(T)| \leq \sqrt{E X^{2}(T)} \leq \sqrt{E(T)}$

Then: $E|W(T)| \leq \sigma^{\prime} \sqrt{E(T)}+\left|\mu^{\prime}\right| E(T)$.

\section{Existence of an Optimal Path}

\section{Definition}

Let $\phi_{n} \in \Phi_{V}(t), n \geq 1$ be a sequence of search plans, we say that $\phi_{n}$ converges to $\phi$ as $\mathrm{n}$ tends to $\infty$ if and only if for any $t \in R^{+}, \phi_{n}$ converges to $\phi(t)$ uniformly on every compact subset.

Note that the set $\Phi_{V}(t)$ constitutes an equicontinuous family of function, also $\left|\phi_{n}(t)\right| \leq V|t|$ for all $n$. We deduce that there exists a subsequence $\phi_{n_{k}}$ which converges to a continuous function $\phi$ by applying the theorem of Asscoli, see [6], it is easy to verify that this function $\phi$ contained in $\Phi_{V}(t)$ that is, the set $\Phi_{V}(t)$ is sequentially compact.

\section{Theorem 4.1}

Let for any $t \in R^{+}, W(t)$ be truncated Brownian process. The mapping

$$
\phi \rightarrow E \tau_{\phi} \in R^{+}
$$

is lower semi continuous on $\Phi_{V}(t)$

Proof let $\mathrm{w}$ be a sample point corresponding to the sample path $\psi(t)$ of $x_{o}+W(t)$

Let $\left(\phi_{n}(t)\right)_{n \geq 1}$ be a sequence of search plans which converges to $\phi \in \Phi_{V}(t)$.

Given $t \in R^{+}$, we define for any $n \geq 1$

$$
B_{n}(t)=\left\{w: \min _{0 \leq x \leq t}\left|\psi(x)-\phi_{n}(x)\right|>a\right\} \text { and } B(t)=\left\{w: \min _{0 \leq x \leq t}|\psi(x)-\phi(x)|>a\right\}
$$

Let $w \in B(t)$ since $\phi_{n(t)}$ converges uniformly on $[0, t]$ to $\phi$, then there exists an integer $n(w)$ such that for any $0 \leq x \leq t$, 


$$
\left|\phi_{n}(x)-\phi(x)\right|<\varepsilon=\frac{1}{2} \min _{0 \leq x \leq t}|\psi(x)-\phi(x)|
$$

Hence for any $0 \leq x \leq t$ and for any $n>n(w)$

$$
\left|\psi(x)-\phi_{n}(x)\right| \geq|\psi(x)-\phi(x)|-\left|\phi(x)-\phi_{n}(x)\right| \geq 2 \varepsilon-\varepsilon=\varepsilon>a
$$

Consequently $w \in B_{n}(t)$ for all $n \geq n(w)$ and hence $B(t) \subset \liminf _{n \rightarrow \infty} B_{n}(t)$. Now, by Fatou's lemma

$$
\int_{0}^{\infty} p(B(t)) \mathrm{d} t \leq \int_{0}^{\infty} p\left(\liminf _{n \rightarrow b} B_{n}(t)\right) \mathrm{d} t \leq \int_{0}^{\infty} \liminf _{n \rightarrow b}\left(B_{n}(t)\right) \mathrm{d} t \leq \liminf _{n \rightarrow b} \int_{0}^{\infty} p\left(B_{n}(t)\right) \mathrm{d} t .
$$

Since sample paths are continuous, then $B_{n}(t)=\left(\tau_{\phi_{n}}>t\right)$ and $B(t)=\left(\tau_{\phi}>t\right)$. It is known that a lower semi-continuous function over the sequentially compact space attains its minimum.

\section{Application}

Let a target moves according to a one-dimensional truncated Brownian motion. In addition, we have a searcher starts the searching from the origin of the line. The searcher moves continuously along its line in both directions of the starting point.We want to calculate $E\left(\tau_{\phi}\right)$ which is given by:

$$
E\left(\tau_{\phi}\right) \leq \lambda(\theta-1)\left[g+(\theta+1)\left(\int_{a}^{0} M(x) v(\mathrm{~d} x)+\int_{0}^{b} B(x) v(\mathrm{~d} x)\right)\right]=\Omega(\lambda, \theta, g, a, b)
$$

Case 1: if:

$$
\begin{gathered}
M_{1}(x)=\sum_{i=1}^{2} \theta^{2 i} p\left(\tilde{\psi}\left(G_{2 i}\right)<-x\right), B_{1}(x)=\sum_{i=1}^{2} \theta^{2 i+1} p\left(\psi\left(G_{2 i+1}\right)>-x\right) \\
g=p\left(\tau_{\phi}>0\right)+\theta p\left(\tau_{\phi}>G_{1}\right)+\theta^{2} \int_{0}^{b} p\left(\psi\left(G_{1}\right)>-x\right) v(\mathrm{~d} x) .
\end{gathered}
$$

Let $X_{0}=x$ be a random variable of initial position of target has a truncated normal distribution, $W(t)=\sigma^{\prime} \sqrt{t} X+\mu^{\prime} t$.

where $X$ is a random variable has a truncated normal, in order to calculate

$$
M_{1}(x)=\sum_{i=1}^{2} \theta^{2 i} p\left(\tilde{\psi}\left(G_{2 i}\right)<-x\right) .
$$

Since $\tilde{\psi}\left(G_{2 i}\right)=W\left(G_{2 i}\right)+c G_{2 i} \quad p\left(W\left(G_{2 i}\right)+c G_{2 i}<-x\right)=p\left(\tilde{\psi}\left(G_{2 i}\right)<-x\right)$

$$
p\left(x<-W\left(G_{2 i}\right)-c G_{2 i}\right)
$$

Since $G_{2 i}=\lambda\left(\theta^{2 i}-1\right), \quad c=\frac{v(\theta-1)}{(\theta+1)}, W\left(G_{2 i}\right)=\sigma^{\prime} \sqrt{G_{2 i}} x+\mu^{\prime} G_{2 i}$

Then we get

$$
\begin{aligned}
& p\left(x<-\sigma^{\prime} \sqrt{\lambda\left(\theta^{2 i}-1\right)} x-\mu^{\prime} \lambda\left(\theta^{2 i}-1\right)-c\left(\lambda\left(\theta^{2 i}-1\right)\right)\right) \\
& =p\left(x+\sigma^{\prime} \sqrt{\lambda\left(\theta^{2 i}-1\right)} x<-\mu^{\prime} \lambda\left(\theta^{2 i}-1\right)-c\left(\lambda\left(\theta^{2 i}-1\right)\right)\right) \\
& =p\left(x\left(1+\sigma^{\prime} \sqrt{\lambda\left(\theta^{2 i}-1\right)}\right)<-\mu^{\prime} \lambda\left(\theta^{2 i}-1\right)-c \lambda\left(\theta^{2 i}-1\right)\right) \\
& =p\left(x<\frac{-\mu^{\prime} \lambda\left(\theta^{2 i}-1\right)-c \lambda\left(\theta^{2 i}-1\right)}{\left(1+\sigma^{\prime} \sqrt{\lambda\left(\theta^{2 i}-1\right)}\right)}\right)=p\left(x<\frac{\lambda\left(\theta^{2 i}-1\right)\left[-\mu^{\prime}-c\right]}{\left(1+\sigma^{\prime} \sqrt{\lambda\left(\theta^{2 i}-1\right)}\right)}\right)
\end{aligned}
$$


Put $\lambda=3, \theta=2, v=10, \mu^{\prime}=2, \sigma^{\prime}=30, c=3.33$. By subsisting in Equation (16)

$$
p\left(x<\frac{\left(3 \times\left(2^{2 i}-1\right)[-2-3.33]\right)}{\left(1+\left(30 \times \sqrt{3\left(2^{2 i}-1\right)}\right)\right)}\right) .
$$

By subsisting (17) in (14) we get:

$$
\begin{aligned}
M_{1}(x) & =\sum_{i=1}^{2} 2^{2 i} p\left(x<\frac{3\left(2^{2 i}-1\right)[-2-3.33]}{\left(1+\left(30 \times \sqrt{3\left(2^{2 i}-1\right)}\right)\right)}\right) \\
& =2^{2} p\left(x<\frac{3\left(2^{2}-1\right)[-2-3.33]}{\left(1+\left(30 \times \sqrt{3\left(2^{2}-1\right)}\right)\right)}\right)+2^{4} p\left(x<\frac{3\left(2^{4}-1\right)[-2-3.33]}{\left(1+\left(30 \times \sqrt{3\left(2^{4}-1\right)}\right)\right)}\right) \\
& =2^{2} p(x<-0.5271)+2^{4} p(x<-1.186) \\
& =2^{2} p\left(\frac{x-\mu^{\prime}}{\sigma^{\prime}}<\frac{-0.5271-2}{30}\right)+2^{4} p\left(\frac{x-\mu^{\prime}}{\sigma^{\prime}}<\frac{-1.186-2}{30}\right) \\
& =2^{2} p(z<-0.08)+2^{4} p(z<-0.1062)=9.168, \text { where } z \sim n(0,1)
\end{aligned}
$$

To calculate $B_{1}(x)$ by the same way we can get:

$$
\begin{gathered}
B_{1}(x)=\sum_{i=1}^{2} \theta^{2 i+1} p\left(\psi\left(G_{2 i+1}\right)>-x\right), \text { since } \psi\left(G_{2 i}\right)=W\left(G_{2 i}\right)-c G_{2 i} . \\
W\left(G_{2 i}\right)=\sigma^{\prime} \sqrt{G_{2 i}} x+\mu^{\prime} G_{2 i}, \quad G_{2 i}=\lambda\left(\theta^{2 i}-1\right), \quad c=\frac{v(\theta-1)}{(\theta+1)}
\end{gathered}
$$

Then: $p\left(\psi\left(G_{2 i}\right)>-x\right)=p\left(W\left(G_{2 i}\right)-c G_{2 i}>-x\right)=p\left(x>-W\left(G_{2 i}\right)+c G_{2 i}\right)$

$$
\begin{aligned}
B_{1}(x) & =\sum_{i=1}^{2} \theta^{2 i+1} p\left(x>\frac{-\mu^{\prime} \lambda\left(\theta^{2 i+1}-1\right)+c \lambda\left(\theta^{2 i+1}-1\right)}{\left(1+\sigma^{\prime} \sqrt{\lambda\left(\theta^{2 i+1}-1\right)}\right)}\right) \\
& =\sum_{i=1}^{2} \theta^{2 i+1} p\left(x>\frac{\lambda\left(\theta^{2 i+1}-1\right)\left[c-\mu^{\prime}\right]}{\left(1+\sigma^{\prime} \sqrt{\lambda\left(\theta^{2 i+1}-1\right)}\right)}\right) \\
& =2^{3} p\left(x<\frac{3\left(2^{3}-1\right)[3.33-2]}{\left(1+\left(30 \times \sqrt{3\left(2^{3}-1\right)}\right)\right)}\right)+2^{5} p\left(x<\frac{3\left(2^{5}-1\right)[3.33-2]}{\left(1+\left(30 \times \sqrt{3\left(2^{5}-1\right)}\right)\right)}\right) \\
& =2^{3} p(x>0.202)+2^{5} p(x>0.426) \\
& =2^{3} p\left(z>\frac{0.202-2}{30}\right)+2^{5} p\left(z>\frac{0.426-2}{30}\right) \\
& =20.8 .
\end{aligned}
$$

Since $E\left(\tau_{\Phi}\right) \leq \lambda(\theta-1)\left[g+\int_{a}^{0} M(x) v(\mathrm{~d} x)+\int_{0}^{b} B(x) v(\mathrm{~d} x)\right]$.

By subsisting of (18), (19) in Equation (20) we can get 


$$
E_{1}\left(\tau_{\Phi}\right) \leq 3\left[g+\int_{a}^{0} 9.168 \mathrm{~d} x+\int_{0}^{b} 20.8 \mathrm{~d} x\right] .
$$

Let $a=-2, b=2$.

Since $g=p\left(\tau_{\Phi}>0\right)+\theta p\left(\tau_{\Phi}>G_{1}\right)+\theta^{2} \int_{0}^{b} p\left(\psi\left(G_{1}\right)>-x\right) v(\mathrm{~d} x)$

$$
\begin{aligned}
p\left(\tau_{\Phi}>G_{2 i+1}\right) \leq & \int_{a}^{0} p\left(x_{0}+W\left(G_{2 i}\right)<H_{2 i} / x_{0}=x\right) v(\mathrm{~d} x) \\
& +\int_{0}^{b} p\left(x_{0}+W\left(G_{2 i+1}\right)>H_{2 i+1} / x_{0}=x\right) v(\mathrm{~d} x) .
\end{aligned}
$$

Put $i=0$ in Equation (22), then we can get

$$
\begin{aligned}
p\left(\tau_{\Phi}>G_{1}\right) \leq & \int_{a}^{0} p\left(x_{0}+W\left(G_{0}\right)<H_{0} / x_{0}=x\right) v(\mathrm{~d} x) \\
& +\int_{0}^{b} p\left(x_{0}+W\left(G_{1}\right)>H_{1} / x_{0}=x\right) v(\mathrm{~d} x) .
\end{aligned}
$$

Since: $H_{i}=(-1)^{i+1} c\left(G_{i}+1+(-1)^{i+1}\right), W\left(G_{i}\right)=\sigma^{\prime} \sqrt{G_{i}} x+\mu^{\prime} G_{i}$, $G_{i}=\lambda\left(\theta^{i}-1\right)$

Then: $H_{0}=(-1) c\left(G_{0}+1-1\right)=-C G_{0}=0, W\left(G_{0}\right)=\sigma^{\prime} \sqrt{G_{0}} x+\mu^{\prime} G_{0}$.

By subsisting of $H_{0}, W\left(G_{0}\right)$ in Equation (23) we can get

$$
p\left(\tau_{\Phi}>G_{1}\right) \leq \int_{a}^{0} p\left(x_{0}<0\right) v(\mathrm{~d} x)+\int_{a}^{b} p\left(x_{0}>H_{1}-W\left(G_{1}\right) / x_{0}=x\right) v(\mathrm{~d} x) .
$$

Since $H_{1}=(-1)^{2} c\left(G_{1}+1+(-1)^{2}\right)=c\left(G_{1}+2\right)=c(\lambda(\theta-1)+2), \quad H_{1}=16.65$ $W\left(G_{1}\right)=\sigma^{\prime} \sqrt{G_{1}} x+\mu^{\prime} G_{1}=51.9615 x+6$.

By subsisting of $H_{1}, W\left(G_{1}\right)$ in Equation (24) we get

$$
\begin{aligned}
p\left(\tau_{\Phi}>G_{1}\right) & \leq \int_{a}^{0} p(x<0) v \mathrm{~d} x+\int_{0}^{b} p(x>16.65-(51.9615 x+6)) \mathrm{d} x \\
& \leq \int_{a}^{0} p(x<0) v \mathrm{~d} x+\int_{0}^{b} p(x+51.9615 x>16.65-6) \mathrm{d} x \\
& \leq \int_{a}^{0} p(x<0)+\int_{0}^{b} p\left(x>\frac{16.65-6}{52.9615}\right) \mathrm{d} x
\end{aligned}
$$

Since $p(x<0)=p(z<-0.07)=0.472$

$p\left(x>\frac{16.65-6}{52.9615}\right)=p(x>0.20)=p\left(\frac{x-\mu^{\prime}}{\sigma^{\prime}}>\frac{0.20-2}{30}\right)=p(z>-0.06)=0.524$.

By subsisting in Equation (25) we can get $p\left(\tau_{\Phi}>G_{1}\right) \leq-a(0.472)+b(0.524)$.

Since $a=-2, b=2, p\left(\tau_{\Phi}>G_{1}\right) \leq 1.992$.

To calculate $p\left(\psi\left(G_{1}\right)>-\chi\right)$, where $\psi\left(G_{2 i+1}\right)=W\left(G_{2 i+1}\right)-c G_{2 i+1}$.

That is, $\psi\left(G_{1}\right)=W\left(G_{1}\right)-c G_{1}$, since $\psi\left(G_{1}\right)=51.9615 x-3.99$

Then

$$
\begin{aligned}
p\left(\psi\left(G_{1}\right)>-x\right) & =p(51.9615 x-3.99>-x) \\
& =p(51.9615 x+x>3.99) \\
& =p(z>-0.06)=0.524 .
\end{aligned}
$$


By subsisting (26), (28) in Equation (13), we get

$$
g=1+2(1.992)+2^{2} \int_{0}^{2} 0.524 \mathrm{~d} z=9.176
$$

By subsisting (29) in (21) we get

$$
E_{1}\left(\tau_{\Phi}\right) \leq 3[9.176+18.336+41.6]=207.336 .
$$

Case 2: if we take: $M_{2}(x)=\sum_{i=1}^{3} \theta^{2 i} p\left(\tilde{\psi}\left(G_{2 i}\right)<-x\right)$ and $B_{2}(x)=\sum_{i=1}^{3} \theta^{2 i+1} p\left(\psi\left(G_{2 i+1}\right)>-x\right)$. By the same way for chosen

$$
\lambda=3, \quad \theta=2, v=10, \mu^{\prime}=2, \sigma^{\prime}=30, c=3.33 .
$$

We can get: $M_{2}(x)=37.42, B_{2}(x)=85.10$.

\begin{tabular}{|c|c|c|c|c|c|c|c|c|c|}
\hline$\lambda$ & $\theta$ & $v$ & $C$ & $\mu^{\prime}$ & $\sigma^{\prime}$ & $g$ & $M_{j}(x)$ & $B_{j}(x)$ & $\Omega()$. \\
\hline \multirow{4}{*}{3} & \multirow{4}{*}{2} & \multirow{4}{*}{10} & \multirow{4}{*}{3.33} & \multirow{4}{*}{0.3} & \multirow{4}{*}{30} & \multirow{4}{*}{8.984} & 9.729 & 19.2158 & 200.6225 \\
\hline & & & & & & & 40.06 & 77.015 & 729.4211 \\
\hline & & & & & & & 155.693 & 281.752 & 2651.619 \\
\hline & & & & & & & 573.24 & 901.032 & 8872.5896 \\
\hline \multirow{4}{*}{3} & \multirow{4}{*}{2} & \multirow{4}{*}{10} & \multirow{4}{*}{3.33} & \multirow{4}{*}{0.5} & \multirow{4}{*}{30} & \multirow{4}{*}{9.016} & 9.665 & 19.384 & 201.34 \\
\hline & & & & & & & 39.7517 & 77.9632 & 733.34 \\
\hline & & & & & & & 154.084 & 287.47 & 2676.40 \\
\hline & & & & & & & 464.1078 & 936.716 & 9031.99 \\
\hline \multirow{4}{*}{3} & \multirow{4}{*}{2} & \multirow{4}{*}{10} & \multirow{4}{*}{3.33} & \multirow{4}{*}{1.5} & \multirow{4}{*}{30} & \multirow{4}{*}{9.138} & 9.347 & 20.2276 & 204.866 \\
\hline & & & & & & & 38.199 & 82.72 & 752.93 \\
\hline & & & & & & & 146.084 & 316.4488 & 2802.6112 \\
\hline & & & & & & & 519.074 & 1123.585 & 9883.365 \\
\hline \multirow{4}{*}{3} & \multirow{4}{*}{2} & \multirow{4}{*}{10} & \multirow{4}{*}{3.33} & \multirow{4}{*}{2} & \multirow{4}{*}{30} & \multirow{4}{*}{9.176} & 9.168 & 20.8 & 206.56 \\
\hline & & & & & & & 37.426 & 85.10 & 762.69 \\
\hline & & & & & & & 142.11 & 331.082 & 2866.71 \\
\hline & & & & & & & 497.02 & 1220.95 & $10,335.41$ \\
\hline \multirow{4}{*}{3} & \multirow{4}{*}{2} & \multirow{4}{*}{10} & \multirow{4}{*}{3.33} & & & & 9.03086 & 21.0704 & 208.0213 \\
\hline & & & & & & & 36.6565 & 88.704 & 772.247 \\
\hline & & & & 2.5 & 30 & 7.256 & 138.174 & 352.896 & 2930.9624 \\
\hline & & & & & & & 475.329 & 1385.088 & $10,798.165$ \\
\hline & & & & & & & 8.8739 & 21.49 & 210.142 \\
\hline & & & & & & & 35.889 & 89.859 & 782.45 \\
\hline 3 & 2 & 10 & 3.33 & 3 & 30 & 9.32 & 134.26 & 360.407 & 2995.97 \\
\hline & & & & & & & 454.034 & 1419.155 & $11,267.1$ \\
\hline
\end{tabular}

Then $E_{2}\left(\tau_{\phi}\right) \leq 762.69$.

Table 1. The upper bound of $E\left(\tau_{\phi}\right)$. 
Case 3: if we take:

$$
M_{3}(x)=\sum_{i=1}^{4} \theta^{2 i} p\left(\tilde{\psi}\left(G_{2 i}\right)<-x\right), \quad B_{3}(x)=\sum_{i=1}^{4} \theta^{2 i+1} p\left(\psi\left(G_{2 i+1}\right)>-x\right) .
$$

By the same way for chosen $\lambda=3, \theta=2, v=10, \mu^{\prime}=2, \sigma^{\prime}=30$, $c=3.33$.

we can get: $M_{3}(x)=142.12, B_{3}(x)=331.082$.

Then $E_{3}\left(\tau_{\phi}\right) \leq 2866.71$.

Case 4: if we take:

$$
M_{4}(x)=\sum_{i=1}^{5} \theta^{2 i} p\left(\tilde{\psi}\left(G_{2 i}\right)<-x\right), \quad B_{4}(x)=\sum_{i=1}^{5} \theta^{2 i+1} p\left(\psi\left(G_{2 i+1}\right)>-x\right) .
$$

By the same way for chosen $\lambda=3, \theta=2, v=10, \mu^{\prime}=2, \sigma^{\prime}=30$, $c=3.33$.

We can get: $M_{4}(x)=497.02, B_{4}(x)=1220.95$.

Then $E_{4}\left(\tau_{\phi}\right) \leq 10335.41$. If we want to see the effect $\mu^{\prime}$ on the search plan, choose $\lambda=3, \theta=2, v=10, \sigma^{\prime}=30, c=3.33$ as constants and give different values to $\mu^{\prime}$, and for each chosen we calculate the values

$B_{j}(x), M_{j}(x), j=1,2,3,4$ and corresponding values of $\Omega($.$) , see Table 1$, in this table we can determine a search plane which make the value of the upper bound of $E\left(\tau_{\phi}\right)$ is small. In future study we can do a program in order to get the search plan for different values of $\lambda, \theta, c, v, \sigma^{\prime}, \mu^{\prime}$.

\section{Conclusion}

In this paper, we investigated the search model for a lost target whose truncated Brownian motion is on a real line, and the expected value of the first meeting between the searcher and target is studied. Also the existence of the optimal search plan that minimizes this expected value is proved. The search model, when the lost target follows truncated Brownian motion on one of finite number of disjoint linear lines will be investigated in the future.

\section{References}

[1] El-Rayes, A.B., Mohamed, A.A. and Abou Gabal, H.M. (2003) Linear Search for a Brownian Target Motion. Acta Mathematica Scientia, 23(B), 321-327.

[2] Mohamed, A., Kassem, M. and El-Hadidy, M.A. (2011) Multiplicative Linear Search for a Brownian Target Motion. Applied Mathematical Modelling, 35, 4127-4139.

[3] El-Hadidy, M.A.A. (2016) Searching for a $d$-Dimensional Brownian Target with Multiple Sensors. International Journal of Mathematics in Operational Research, 9 , 279-301. https://doi.org/10.1504/IJMOR.2016.078822

[4] Elrayes, A.B. and Abd El-Moneim, M.A. (1989) Searching for a Randomly Moving Target. The Third ORMA Conference Egypt, 2, 323-329.

[5] Feller, W. (1966) An Introduction to Probability Theory and Its Applications. Willey, New York.

[6] Royden, H.L. (1968) Real Analysis. Second Edition, Macmillan, London. 
Submit or recommend next manuscript to SCIRP and we will provide best service for you:

Accepting pre-submission inquiries through Email, Facebook, LinkedIn, Twitter, etc. A wide selection of journals (inclusive of 9 subjects, more than 200 journals)

Providing 24-hour high-quality service

User-friendly online submission system

Fair and swift peer-review system

Efficient typesetting and proofreading procedure

Display of the result of downloads and visits, as well as the number of cited articles Maximum dissemination of your research work

Submit your manuscript at: http://papersubmission.scirp.org/

Or contact am@scirp.org 